\title{
Evolução da hipermetropia na infância
}

\author{
Evolution of hyperopia in childhood
}

\author{
Geraldo de Barros Ribeiro ${ }^{1}$ \\ Roberto Martins Gonçalves ${ }^{2}$ \\ Cristiano Menezes Diniz ${ }^{2}$ \\ Simone Teixeira de Paula ${ }^{3}$ \\ Henderson Celestino de Almeida ${ }^{4}$
}

\section{RESUMO}

Objetivo: Avaliar a evolução da hipermetropia na infância. Métodos: Estudo retrospectivo, pela análise de prontuários de 67 pacientes que tiveram seu primeiro exame refrativo até os 3 anos de idade e tendo um período mínimo de seguimento de 5 anos. A idade média ao primeiro exame foi 18,5 $\pm 6,9$ meses e a idade média final de acompanhamento foi de $8,4 \pm$ 1,7 anos, o que proporcionou um tempo médio de seguimento dos pacientes de 6,8 \pm 1,3 anos. Resultados: Houve aumento da hipermetropia quando comparado o $1^{\circ} \operatorname{com~o} 3^{\circ}$ ano de vida $(\mathrm{p}<0,05)$, mantendo após esse período uma curva sem diferenças estatísticas até o $10^{\circ}$ ano de vida. Não houve diferença na variação da hipermetropia quando se dividiram as crianças em grupos com baixas e altas ametropias. Conclusão: O presente estudo demonstra que a hipermetropia apresenta pequena variação nos primeiros anos de vida, porém tende a manter-se estatisticamente inalterada dos 3 aos 10 anos de idade.

Descritores: Hiperopia; Criança; Estudos retrospectivos; Evolução

\section{INTRODUÇ̃̃̃O}

A hipermetropia é a ametropia mais encontrada em crianças em nosso meio $^{(1-3)}$. Dados da literatura ${ }^{(4)}$ mostram que a hipermetropia pode acometer $55 \%$ da população sendo que, na maior parte dos casos, trata-se de grau inferior a $0,45 \mathrm{D}$, portanto, assintomática. Alguns autores acreditam que a grande maioria dos recém-nascidos é hipermétrope e que, com o passar do

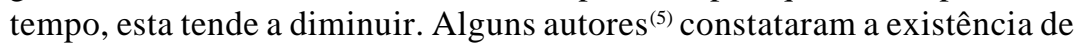
hipermetropia em $80 \%$ dos recém-nascidos, de emetropia em $15 \%$ e de miopia em $5 \%$, enquanto outros ${ }^{(6)}$ acreditam que todas as crianças nascem hipermétropes.

O crescimento do globo ocular, as alterações na curvatura corneana e no poder dióptrico do cristalino promovem mudanças na refração. O aumento axial do olho da infância até a idade adulta é de aproximadamente $7 \mathrm{~mm}$, o que necessitaria de uma redução de 30 dioptrias no poder refrativo para manter o estado de emetropia ${ }^{(7)}$. As medidas ceratométricas, que são em média de 51,2 dioptrias ao nascimento, reduz-se principalmente nos primeiros 6 meses de vida, mantendo-se praticamente inalterada com o crescimento $^{(8)}$. O poder refrativo do cristalino reduz em média 8 dioptrias no primeiro ano de vida. Esta redução mantém-se, porém mais lentamente, até o final os 6 a 7 anos, quando estabiliza até a idade adulta ${ }^{(8)}$.

A evolução do erro refrativo, em especial da hipermetropia, na infância tem sido tema de estudo de vários trabalhos científicos ${ }^{(8-13)}$. Alguns autores constataram um aumento da hipermetropia nos primeiros anos de vida ${ }^{(10,14)}$, enquanto outros não observaram essa tendência ${ }^{(6,8)}$. Como indivíduos com elevado grau de hipermetropia possuem maior probabilidade de desenvol- 
verem estrabismo e ambliopia ${ }^{(15-16)}$, é fundamental que esse erro refrativo seja identificado de forma mais precisa possível e que saibamos como ele evolui durante o período de desenvolvimento visual.

O presente estudo visa avaliar a evolução da hipermetropia na infância, com o objetivo de ajudar na compreensão do complexo processo de emetropização.

\section{MÉTODOS}

O trabalho foi realizado de forma retrospectiva, analisando-se prontuários de crianças atendidas nos serviços de Estrabismo dos Hospitais São Geraldo e Infantil São Camilo em Belo Horizonte, Minas Gerias, entre os anos de 1970 e 2001, e que tiveram seu primeiro exame com, no máximo, 36 meses de idade. Foram selecionados 112 prontuários. Estes pacientes deveriam ter sido acompanhados por no mínimo cinco anos e submetidos a pelo menos três consultas oftalmológicas. Indivíduos portadores de miopia ou anisometropia acima de 1D foram excluídos do estudo. Foi considerado, também, como critério de exclusão do estudo, qualquer grau de ambliopia que fosse observado durante o acompanhamento do paciente. Sessenta e sete pacientes preencheram todos os critérios. A idade mínima ao primeiro exame foi de 4,2 meses e a máxima de 34,5 meses, com média de 18,5 $\pm 6,9$ e mediana de 18,7 meses. A idade final de acompanhamento variou de 5 a 10 anos com média de $8,4 \pm 1,7$ o que proporcionou um tempo médio de seguimento dos pacientes de $6,8 \pm 1,8$ anos. O número de consultas variou de 3 a 8 com média de 5,2 $\pm 1,3$.

O exame refrativo foi realizado sob cicloplegia: uma gota de colírio anestésico, cloridrato de proximetacaína, e logo em seguida uma gota de ciclopentolato seguidas após 5 minutos de uma gota de tropicamida. Após quarenta minutos da instilação do primeiro colírio, a retinoscopia foi realizada por apenas dois dos pesquisadores (H.C.A. ou G.B.R.).

Os dados foram computados e analisados através de um banco de dados do programa Epiinfo 6.04 (CDC - Atlanta).

\section{RESULTADOS}

A menor hipermetropia ao exame inicial observada entre as 67 crianças analisadas foi de $+0,50 \mathrm{D}$ e a maior, $+8,00 \mathrm{D}$. A análise da evolução da ametropia está disposta nos gráficos 1 , 2 e 3 . Os valores mostrados se relacionam à média entre os olhos, ou seja, representam valores de ambos os olhos. Tal medida foi tomada com a finalidade de facilitar as análises, uma vez que se observou não existir diferença significativa entre as refrações de cada olho separadamente.

No gráfico 1 , nota-se que a diferença, ou seja, o aumento da hipermetropia em relação ao primeiro ano se tornou estatisticamente significativa a partir do $3^{\circ}$ ano, mantendo-se desta forma até o $8^{\circ}$ ano de idade. Entretanto, não houve diferença significativa entre os valores encontrados no $2^{\circ}$ ano e nos

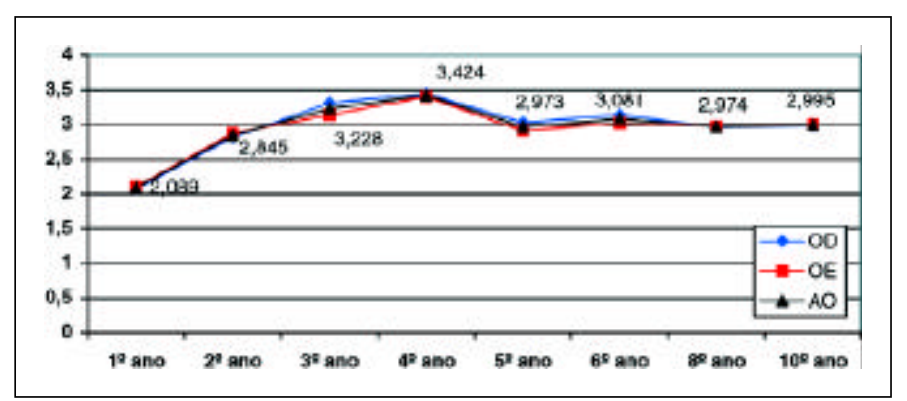

Gráfico 1 - Média da hipermetropia encontrada de acordo com o ano (idade) de avaliação ( $n=67$ )

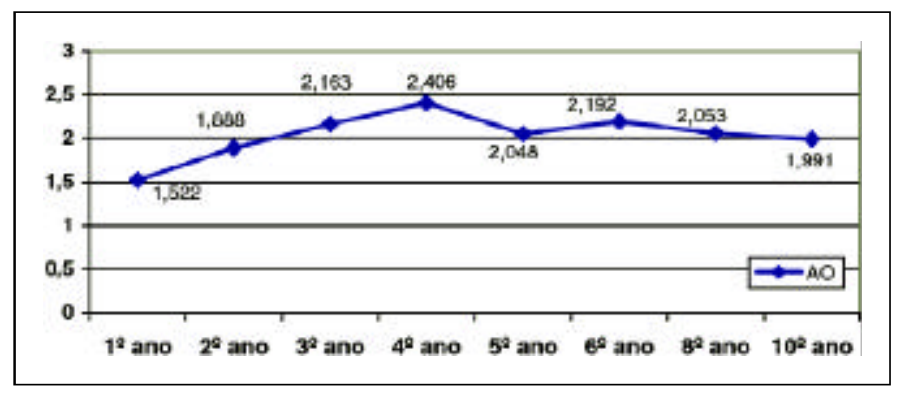

Gráfico 2 - Média da hipermetropia em ambos os olhos de acordo com o ano (idade) de avaliação, nos pacientes com ametropia inicial menor ou igual a $+3,00 D(n=49)$

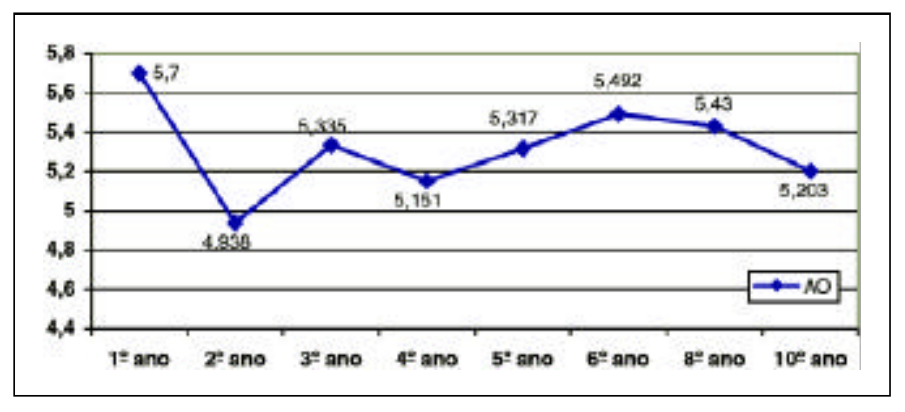

Gráfico 3 - Média da hipermetropia em ambos os olhos de acordo com o ano (idade) de avaliação, nos pacientes com ametropia inicial maior que $+3,00 \quad D(n=18)$

anos subseqüentes. A curva, como um todo, também não se mostrou estatisticamente significativa apresentando, na comparação das médias, um valor de $\mathrm{p}>0,05$.

Ao serem separadas as hipermetropias iniciais em dois grupos, acima e abaixo de $+3,00 \mathrm{D}$, foi possível observar que, em ambos os grupos, as curvas não apresentaram variação estatisticamente significativa (Gráficos 2 e 3).

\section{DISCUSSÃO}

Os dados coletados neste estudo demonstram que a hipermetropia apresenta uma pequena elevação nos primeiros anos de vida, o que também foi observado por outros autores ${ }^{(10,14)}$. Vários autores observaram que as crianças são geralmente hi- 
permétropes e que tendem a evoluir para emetropização com o

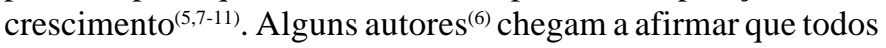
os recém-nascidos são hipermétropes e que, com o passar do tempo, essa hipermetropia tende a diminuir. Em um trabalho recente $^{(11)}$, mostrou-se substancial redução da hipermetropia durante o segundo e terceiro anos de vida e que o ritmo de redução possui relação linear com o poder da ametropia inicial.

Em estudo nacional, analisou-se a evolução da hipermetropia do nascimento aos dezoito anos em indivíduos ortotrópicos e estrábicos ${ }^{(9)}$. Formaram-se 5 grupos com idades que variavam de 0 a 2 anos, 4 a 6 anos, 8 a 10 anos, 12 a 14 anos e de 16 a 18 anos. Nosso estudo foi realizado em crianças com até 10 anos de idade, porém com seguimento mínimo de 5 anos. Observouse, no referido trabalho ${ }^{(9)}$, que a hipermetropia diminuiu significativamente na adolescência, sendo que o decréscimo iniciou mais precocemente nos indivíduos não estrábicos, de acordo com resultado encontrado na literatura científica internacional $^{(10)}$. Entretanto, neste estudo internacional, constatou-se aumento da hipermetropia entre o nascimento e os 7 anos de idade, redução nos 8 anos seguintes e uma lenta diminuição durante os 15 anos subseqüentes. A explicação desse autor para o aumento da hipermetropia na primeira faixa etária analisada foi a diminuição da curvatura da córnea ou do cristalino. $\mathrm{O}$ aumento da hipermetropia nos primeiros anos de vida corrobora com os resultados de outro trabalho ${ }^{(14)}$.

Quanto à evolução da hipermetropia em crianças, este estudo mostrou uma curva praticamente regular, com pequeno aumento do $1^{\circ}$ para o $10^{\circ}$ ano de idade. A diferença tornou-se significativa $(\mathrm{p}<0,05)$ ao compararem-se os valores do $1^{\circ}$ ano com os do $3^{\circ}$ e $8^{\circ}$ anos. Bicas ${ }^{(17)}$ salienta que, numa segunda refratometria, a regra é o encontro de valores discretamente mais altos do que na primeira, fato esse que não depende da droga cicloplégica inicialmente usada e sim do relaxamento do músculo ciliar provocado pela correção óptica do vício refrativo durante certo tempo. Além disto, crianças mais novas choram mais, durante a instilação dos colírios e as lágrimas seguramente diluem as drogas instiladas, reduzindo sua absorção e conseqüentemente seu efeito. Isto também explica o freqüente aumento da hipermetropia no $2^{\circ}$ e $3^{\circ}$ exames. Sendo assim, quando a análise foi realizada a partir do $2^{\circ}$ ano, encontrou-se discreto aumento da hipermetropia, não significativo, mas que foi suficiente para invalidar toda a importância das diferenças com os anos subseqüentes, ou seja, os valores de "p" tornaram-se maiores que 0,05 .

Se a hipermetropia total deve ser corrigida nas crianças com esotropia, existem dúvidas na literatura sobre quando e quanto receitar para crianças com visão binocular normal. Alguns autores ${ }^{(15)}$ analisaram prontuários de 5.042 crianças e observaram que as que possuíam hipermetropias acima de 2,76 tinham uma probabilidade 4 vezes maior de possuir estrabismo ou ambliopia. Os autores acreditam que até 3 dioptrias esféricas positivas em crianças ortotrópicas não devam ser corrigidas. Todavia, os dados encontrados neste estudo demonstram que a hipermetropia não tende a diminuir durante a infância, justamente durante o período de desenvolvimento visual e plasticidade sensorial; portanto, devemos estar alertas quanto a uma possível ambliopia ou sintomas de baixa concentração em leituras prolongadas, causadas por hipermetropia elevada.

\section{CONCLUSÃO}

O papel da hipermetropia como importante indutor de ambliopia já é bem conhecido. Além disso, como os vícios refrativos têm influência significativa no estrabismo, é importante sua determinação precoce e de maneira acurada. Acredita-se que tal ametropia deva ser corrigida na totalidade, logo que diagnosticada em pacientes esotrópicos com componente acomodativo importante. A análise dos pacientes estudados evidenciou que hipermetropia varia nos primeiros anos de vida, porém não parece haver redução dos valores dióptricos com o passar dos anos durante a infância.

\section{ABSTRACT}

Purpose: To study the evolution of hyperopia during childhood. Methods: A retrospective study through the analysis of 67 patients' charts. All patients were checked before the $3^{\text {rd }}$ year of age and were followed for at least 5 years. The mean age at the first examination was $18.5 \pm 6.9$ months and the mean age at the end of the follow-up was $8.4 \pm 1.7$ years, providing an average time of the $6.8 \pm 1.8$ years of follow-up. Results: There was an increase in hyperopia when comparing the $1^{\text {st }}$ with the $3^{\text {rd }}$ year of life $(\mathrm{p}<0.05)$. This was maintained without statistically significant variation until the $10^{\text {th }}$ year of life. There was no difference in the variation of the hyperopia when the children were separeted into groups with low and high ametropia. Conclusion: The present study demonstrates that hyperopia presents small variations during the first years of life, however it tends to be statistically stable from the $3^{\text {rd }}$ to the $10^{\text {th }}$ year of age.

Keywords: Hyperopia; Children; Retrospective studies; Evolution

\section{REFERÊNCIAS}

1. Prado D. Noções de óptica, refração ocular e adaptação de óculos. $4^{\mathrm{a}}$ ed. Rio de Janeiro, Edigraf; 1963. p.160.

2. Del Rio EG. Óptica fisiológica clínica. 2a ed. Barcelona, Toray; 1972. p.394.

3. Kwitko S, Kwitko II. Casuística dos erros de refração no Hospital das Clínicas de Porto Alegre. Rev AMRIGS 1984;28:318-24.

4. Geraissate E. Hipermetropia. Arq Bras Oftalmol 2000;63:499-501.

5. Vaughan DG, Cook R, Asbury T. Oftalmologia general. $2^{\text {a }}$ ed., Mexico, El Manual Moderno; 1970. p.284p

6. Lyle TK. Worth and Chavasse's Squint. London: Baillière, Tindall and Cos; 1950. p. 139 .

7. Bennett AG, Francis JL. Ametropia and its correction. In: Davson H, editor. The eye. New York, Academic Press; 1962. p.145.

8. Gordon RA, Donzis PB. Refractive development of human eye. Arch Ophthalmol 1985;103:785-9. 
9. Silva MHA, Araújo JS, Souza-Dias CR. Evolução da hipermetropia na infância e adolescência. Arq Bras Oftalmol 1984;47:146-53.

10. Brown EV. Net average yearly changes in refraction of atropinized eyes from birth to beyond middle life. Arch Ophthalmol 1938;19:719-35.

11. Atkinson J, Anker S, Bobier W, Braddick O, Durden K, Nardini W, et al Normal emmetropization in infants with spectacle correction for hyperopia. Invest Ophthalmol Vis Sci 2000;41:3726-31.

12. Porto CC. Semiologia médica. $2^{a}$ ed. Rio de Janeiro. Guanabara Koogan; 1994. p.12-14;169-202
13. Ehrlich DL, Braddick OJ, Atkinson J, Anker S, Weeks F, Hartley T, et al Infant emmetropization: a longitudinal study of refraction components from 9 months of age. Optom Vis Sci 1997;74:822-43.

14. Wortham C. The increase in hyperopia in children. Br Orthopt J 1978;35:83-90.

15. Fulton AB, Dobson V, Salem D, Mar C, Petersen RA, Hansen RM. Cycloplegic refraction in infants and young children. Am J Ophthalmol 1980;90:239-47.

16. Burian H, Von Noorden GK. Binocular Vision and ocular motility. St. Louis The C. V. Mosby; 1980. p.173.

17. Bicas HEA, Nóbrega JFC. Porque usar ciclopentolato para exame refratométrico em estrábicos. Rev Bras Oftalmol 1974;33:135.

\title{
XI Simpósio Infernacional de Córnea Lentes de Contato - UNICAMP
}

\author{
26 e 27 de Março de 2004 \\ Grand Hyatt Hotel - Sá̋ Paulo
}

\section{Pré-Simpósio: 22 a 25 de Março de 2004 \\ Locais: Hospital das Clínicas da UNICAMP - Campinas e Grand Hyatt Hotel - São Paulo - SP}

INSCRIÇÕESE INFORMAÇÕES: Agência CreativeSolution

Tel.: (11) 5539-2186

Fax: (1 1) 5038-7460

E-mail:simposiounicamp@terra.com.br 